\title{
BMJ Open Associations between sleep quality and its domains and insufficient physical activity in a large sample of Croatian young adults: a cross-sectional study
}

\author{
Lovro Štefan, ${ }^{1}$ Goran Sporiš, ${ }^{1}$ Tomislav Krističević, ${ }^{2}$ Damir Knjaz ${ }^{2}$
}

To cite: Štefan L, Sporiš G, Krističević T, et al.

Associations between sleep quality and its domains and insufficient physical activity in a large sample of Croatian young adults: a crosssectional study. BMJ Open 2018;8:e21902. doi:10.1136/ bmjopen-2018-021902

- Prepublication history for this paper is available online To view these files, please visit the journal online (http://dx.doi. org/10.1136/bmjopen-2018021902).

Received 24 January 2018 Revised 19 June 2018 Accepted 22 June 2018

\section{Check for updates}

(c) Author(s) (or their employer(s)) 2018. Re-use permitted under CC BY-NC. No commercial re-use. See rights and permissions. Published by BMJ.

${ }^{1}$ Department of General and Applied Kinesiology, Faculty of Kinesiology, University of Zagreb, Zagreb, Croatia ${ }^{2}$ Department of Sports Kinesiology, Faculty of Kinesiology, University of Zagreb, Zagreb, Croatia

Correspondence to Mr. Lovro Štefan; lovro.stefan1510@gmail.com

\section{ABSTRACT}

Objectives The main purpose of the present study was to explore the associations between sleep quality and insufficient physical activity.

Design Cross-sectional.

Setting Faculties in Croatia.

Participants 2100 university students (1049 men and 1051 women) aged 18-24 years were recruited.

Primary outcome To assess the domains of sleep quality (independent variables) and 'insufficient' physical activity (dependent variable), we used previously validated Pittsburgh Sleep Quality Index and International Physical Activity questionnaires. Logistic regressions were used to calculate the associations between the sleep quality and 'insufficient' physical activity.

Results When sleep quality domains were entered separately into the model, very bad subjective sleep quality (OR $3.09 ; 95 \% \mathrm{Cl} 1.50$ to 6.56 ), $>60$ min of sleep latency (OR 2.17; $95 \% \mathrm{Cl} 1.39$ to 3.39$),<7$ hours of sleep (OR 1.56; $95 \% \mathrm{Cl} 1.24$ to 1.96$),<65 \%$ of habitual sleep efficiency (OR 2.26; $95 \% \mathrm{Cl} 1.26$ to 4.05 ), sleep disturbances $>1$ /week (OR 1.61; $95 \% \mathrm{Cl} 1.03$ to 2.52), use of sleep medication $>1$ /week (OR $3.35 ; 95 \% \mathrm{Cl} 1.83$ to 6.10$)$, very big daytime dysfunction problem (OR 2.78; $95 \% \mathrm{Cl} 1.57$ to 4.93$)$ and poor sleep quality $(1.53 ; 95 \% \mathrm{Cl}$ 1.23 to 1.91) were associated with 'insufficient' physical activity. When all sleep quality domains were entered simultaneously into the model, the same significant associations remained, except for sleep disturbances. Both models were adjusted for gender, body mass index, self-rated health, life satisfaction, socioeconomic status, presence or absence of chronic diseases, smoking status, binge drinking and psychological distress.

Conclusions Our results show that 'poor' sleep quality is associated with 'insufficient' physical activity in young adults. In order to improve, special strategies and policies that leverage 'good sleep' quality are warranted.

\section{INTRODUCTION}

Physical inactivity has become a major public health problem worldwide. It is a term used to identify people who do not meet the recommendations of participating in regular physical activity. For people aged 18-65 years, $\mathrm{WHO}^{1}$ stated that $150 \mathrm{~min} /$ week of moderate

\section{Strengths and limitations of this study}

This is one of the first studies exploring the associations between sleep quality and 'insufficient' physical activity in a large sample of young adults $(n=2100)$.

- Results showed that 'poor sleep' quality was associated with 'insufficient' physical activity.

- We used subjective measures (questionnaires) to assess both dependent and independent variables, which might have led to potential bias.

Due to a cross-sectional design, we cannot establish the direction of the associations.

or $75 \mathrm{~min} /$ week of vigorous physical activity is the minimum level of physical activity required to achieve health benefits. Unfortunately, $21 \%$ of people aged 15 years and older are physically inactive, with higher prevalence among women and in most developed countries. ${ }^{2}$ It has been well documented that physical inactivity leads to many cardiovascular, metabolic, musculoskeletal and mental diseases and is associated with overall mortality. $^{34}$

Among numerous covariates, ${ }^{5}$ sleep quality can also influence physical activity, especially in young adults. ${ }^{6}$ Sleep quality is defined as 'one's perception that they fall asleep easily, get sufficient duration so as to wake up feeling rested, and can make it through their day without experiencing excessive daytime sleepiness.' ${ }^{,}$Although numerous studies have shown the associations between physical activity and sleep quality, ${ }^{4-689}$ little is known about the reverse association, that is, sleep quality is associated with physical activity. Specifically, Lentino et $a l^{10}$ showed that in a sample of 14148 (83.4\% men) National Military Guard members, 'poor' sleepers were significantly less likely than 'good' sleepers to meet aerobic exercise and resistance training recommendations and 
pass their army physical fitness test. In one longitudinal study, authors showed that better initial sleep quality predicted higher levels of later physical activity beyond the effects of prior physical activity, while initial physical activity did not predict later sleep quality after adjusting for prior sleep quality in a sample of older adults. ${ }^{11}$ In general, a recent systematic review has shown that both sleep and physical activity influence each other through physiological and psychological mechanisms and that the association is bidirectional. ${ }^{12}$ Based on the available literature, there has been lacking of studies examining the associations between 'poor' sleep quality and its domains and 'insufficient' physical activity in young adults. Young adults generally sleep less, compared with decades ago, ${ }^{13}$ and go through big lifestyle changes, such as engaging in extensive electronic media use, academic demands, having a family or starting to work, which could potentially lead to 'insufficient' physical activity. ${ }^{14}$ Also, gender differences in poor sleep quality have been previously reported in young adults. ${ }^{15}$ In general, Fatima et $a l^{15}$ showed that more than half of the study participants were found to have poor sleep quality with slightly higher prevalence among women. However, another study showed no gender differences in sleep quality after adjusting for sociodemographic, lifestyle and psychological factors. ${ }^{16}$

Therefore, the main purpose of the present study was to explore the associations between sleep quality and its domains and 'insufficient' physical activity in a large sample of young adults. We hypothesised that 'poor sleep quality' would be associated with 'insufficient' physical activity.

\section{MATERIALS AND METHODS}

\section{Patient and public involvement}

At the beginning, we presented our participants the main problems of the associations between 'poor' sleep quality and 'insufficient' physical activity, especially in young adults. Only the researchers performed the study and none of our participants were involved in the recruitment to, and conduct of, the study. Our participants were involved via teachers working at faculties and emails with detailed description of the study project with clear aims and hypothesis. By completing the study, all participants were told that they have free data access by request and those data will be sent to them via email. However, all the procedures in this study were anonymous.

\section{Participants}

This cross-sectional study was conducted between September and October 2017 among university students in Zagreb, the capital city of Croatia, with approximately 1000000 citizens. The University of Zagreb is composed of 33 faculties (departments) and between 65000 and 70 000 attend the University every year. A random sampling approach was used to select faculties. At the first stage, we randomly selected 8 out of 33 faculties. At the second stage, we contacted teachers from each faculty to help us organise the sampling procedure. A recruitment announcement was sent via emails and e-newsletter to the teachers with a request to pass the study information to students. All eight faculties agreed to take part in the study, representing 2320 students enrolled in the 2017 academic year. Of these, 2100 students (1041 men and 1059 women, aged 18-24 years) provided full data $(90.5 \%)$ and were enrolled in further analysis. Students came from a variety of social (psychology, political sciences, economy and business), technical (computing, information technologies, electrical engineering, civil engineering, mechanical engineering, graphic arts and naval architecture) and health-related (medical doctors, physiotherapists, nurses) sciences. We followed the methods from previously published studies conducted in the same sample. ${ }^{17-19}$ Before the main analysis, we examined the differences between the particpants who provided completed data and non-valid participants in terms of age, the proportion of gender, body mass index and self-rated health. No significant differences were observed and no potential bias was made $(\mathrm{p}=0.21-0.74)$. All the analyses and procedures were anonymous and in accordance with the Declaration of Helsinki and approved by the Institutional Review Board of the Faculty of Kinesiology (Ethics Code No: 16/2017). Also, all participants gave their written informed consent for participation in the study.

\section{Outcome variable}

To assess physical activity in the last 7 days, we used International Physical Activity questionnaire, a reliable and valid instrument designed to measure physical activity in respondents between ages 18 and $65 .{ }^{20}$ It provided information about the time and number of days spent in light, moderate and vigorous intensity physical activity. For each participant, we calculated the time spent in moderate and vigorous physical activity. According to the $\mathrm{WHO},{ }^{1}$ sufficient physical activity is defined as doing at least (1) $150 \mathrm{~min} /$ week of moderate physical activity or (2) $75 \mathrm{~min} /$ week of vigorous physical activity or (3) an equivalent combination of both. Thus, we categorised the participants who met the aforementioned recommendation as sufficiently active compared with the participants who did not meet the recommended levels of physical activity weekly.

\section{Independent variables}

To assess sleep quality, we used Pittsburgh Sleep Quality Index (PSQI), a highly reliable and valid instrument specifically designed to measure sleep quality. ${ }^{21}$ It is composed of 19 questions, which create seven major components. Each component is scored from 0 to 3 points, where lower point denotes no problems, while higher score denotes worsening problems in following order: (1) subjective sleep quality (very good vs very bad), (2) sleep latency ( $\leq 15 \mathrm{~min}$ to $>60 \mathrm{~min}$ ), (3) sleep duration ( $\geq 7$ hours to $<5$ hours), (4) sleep efficiency ( $\geq 85 \%$ to $<65 \%$ hours sleep/hours in bed), (5) sleep 
disturbances (not during the past month to $\geq 3$ times per week), (6) use of sleeping medications (none to $\geq 3$ times a week) and (7) daytime dysfunction (not a problem to a very big problem). All seven components are then summed up to create a scale from 0 to 21 points. For the purpose of the present study, we used both seven components separately and final score dichotomised into two categories: (1) $\leq 5$ (good sleep quality) vs $(2)>5$ (poor sleep quality). ${ }^{21}$ The reliability of the PSQI in our study was satisfactory (Cronbach's $\alpha=0.73$ ).

\section{Covariates}

Self-rated health was assessed using one-item question: 'How would you rate your health?' Answers were arranged along a Likert-type scale as follows: (1) very poor, (2) poor, (3) fair, (4) good and (5) excellent. This measure has previously been shown as reliable and valid. ${ }^{22}$ For the purpose of this study, we dichotomised the outcome variable into good (fair, good and excellent) versus poor (poor and very poor) self-rated health. ${ }^{23}$ Participants self-reported their height in metres $(\mathrm{m})$ and weight in kilograms $(\mathrm{kg})$, from which body mass index $\left(\mathrm{kg} / \mathrm{m}^{2}\right)$ was calculated. Before the study began, we had chosen 35 men and 40 women to validate self-reported height and weight with the objective measure taken by trained survey staff. Pearson's coefficient of correlation showed excellent relationship between two measures in men $(r=0.96)$ and women $(r=0.97)$. For the purpose of this study, we divided body mass index score into two categories: (1) normal $\left(<25 \mathrm{~kg} / \mathrm{m}^{2}\right)$ and (2) overweight/obesity $\left(\geq 25.0 \mathrm{~kg} / \mathrm{m}^{2}\right)$. Socioeconomic status was assessed by one-item question: 'How would you perceive your socioeconomic status?' Responses were arranged along three-item scale as follows: (1) below average, (2) average and (3) above average. Smoking status was categorised as: (1) non-smoker, (2) former smoker and (3) present smoker. Binge alcohol consumption was assessed by one-item question: 'How often do you have (for men) 5 or more and (for women) 4 or more drinks on one occasion? ${ }^{24}$ Those who had (for men) five or more and (for women) four or more drinks on one occasion were categorised as Yes, compared with No group who had less drinks on one occasion. Life satisfaction was assessed using one-item question: 'How would you rate your life satisfaction?' Answers were arranged along a Likert-type scale as follows: (1) very unsatisfied, (2) unsatisfied, (3) fair, (4) satisfied and (5) very satisfied. This measure has previously been shown as reliable and valid. ${ }^{25}$ For the purpose of this study, we dichotomised the outcome variable into good (fair, satisfied and very satisfied) versus poor (unsatisfied and very unsatisfied) life satisfaction. ${ }^{25}$ The presence or absence of a chronic disease was asked by one-item question: 'Have you ever been told by a doctor, that you suffer from any kind of chronic disease?' with Yes and No answers. Psychological distress was assessed by using Kessler's six-item questionnaire: (1) 'How often during the past 30 days did you feel nervous?', (2) 'How often during the past 30 days did you feel hopeless?', (3)
'How often during the past 30 days did you feel restless or fidgety?', (4) 'How often during the past 30 days did you feel so depressed that nothing could cheer you up?', (5) 'How often during the past 30 days did you feel that everything was an effort?' and (6) 'How often during the past 30 days did you feel worthless? ${ }^{26}$ Each question is scored from 0 (none of the time) to 4 (all of the time). Scores of each question are summed up between 0 and 24, with lower score indicating lower level of psychological distress. Kessler $e t a l^{26}$ showed that responses $<13$ points vs $\geq 13$ points discriminated participants without and with psychological distress.

\section{Data analysis}

Basic descriptive statistics of the study participants are presented as frequencies (n) and percentages (\%). Differences between categorical variables were analysed using $\mathrm{X}^{2}$ test. To examine the associations between 'poor' sleep quality with 'insufficient' physical activity, we used multiple logistic regression analysis. We calculated ORs with $95 \%$ CIs by using contrast subcommand. We tested the data for multicollinearity using the variance inflation factors, normality of residuals using the normal probability plot and histogram of residuals and heteroscedasticity using the standardised residuals versus predicted plot. The variance inflation factors in our model ranged from 1.01 to 1.85 indicating no multicollinearity and the other assumptions were also met. In univariate model, we examined the associations between 'poor' sleep quality and its domains and 'insufficient' physical activity. In multivariate model, we examined the associations between 'poor' sleep quality and its domains and 'insufficient' physical activity after adjusting for body mass index, self-rated health, life satisfaction, socioeconomic status, smoking status, alcohol consumption, presence or absence of chronic diseases and psychological distress. In both models, sleep duration was categorised as $>7$ hours vs $\leq 7$ hours of sleep, due to no response in categories '5-6hours' and ' $<5$ hours'. Also, due to a very small number of participants reported having sleep disturbances and consuming sleep medication for $\geq 3$ /week, we summed up ' $\geq 3$ / week' category with ' $1-2$ /week' category in both variables. The interaction term between gender and sleep quality domains was not statistically significant and we dropped the gender-stratified analyses. Significance was set up at $\alpha=0.05$ and it was one sided. All the analyses were performed in Statistical Package for Social Sciences Software, V.22 (IBM).

\section{RESULTS}

Basic descriptive statistics of the study participants are presented in table 1. In general, higher percentage of 'sufficiently active' participants reported very good subjective sleep quality $(\mathrm{p}<0.001), \leq 15 \mathrm{~min}$ of sleep latency $(p<0.001)$, sleeping $>7$ hours per night $(p<0.001)$, having higher percentage of habitual sleep efficiency $(\mathrm{p}=0.002)$, having no sleep disturbances $(\mathrm{p}<0.001)$ and 
Table 1 Characteristics of the study participants, stratified by gender (Croatia, 2017)

\begin{tabular}{|c|c|c|c|c|}
\hline \multirow[b]{2}{*}{ Study variables } & \multirow{2}{*}{$\begin{array}{c}\begin{array}{l}\text { Total sample } \\
(\mathrm{n}=2100)\end{array} \\
\mathrm{n}(\%) \\
\end{array}$} & \multirow{2}{*}{$\begin{array}{l}\begin{array}{l}\text { Men } \\
(n=1041)\end{array} \\
n(\%)\end{array}$} & \multirow{2}{*}{$\begin{array}{l}\begin{array}{l}\text { Women } \\
(n=1059)\end{array} \\
n(\%) \\
\end{array}$} & \multirow[b]{2}{*}{ P values* } \\
\hline & & & & \\
\hline \multicolumn{5}{|c|}{ Subjective sleep quality } \\
\hline Very good & $687(32.7)$ & $360(34.6)$ & $327(30.9)$ & \\
\hline Fairly good & 1179 (56.1) & $577(55.4)$ & $602(56.8)$ & \\
\hline Fairly bad & $204(9.7)$ & $92(8.8)$ & $112(10.6)$ & \\
\hline Very bad & $30(1.4)$ & $12(1.2)$ & $18(1.7)$ & 0.163 \\
\hline \multicolumn{5}{|l|}{ Sleep latency (min) } \\
\hline$\leq 15$ & $292(13.9)$ & $160(15.4)$ & $132(12.5)$ & \\
\hline $16-30$ & $868(41.3)$ & (450 (43.2) & $418(39.5)$ & \\
\hline $31-60$ & 769 (36.6) & $359(34.5)$ & $410(38.7)$ & \\
\hline$>60$ & $171(8.1)$ & $72(6.9)$ & $99(9.3)$ & 0.010 \\
\hline \multicolumn{5}{|c|}{ Sleep duration (hours) } \\
\hline$>7$ & 1615 (76.9) & $824(79.2)$ & $791(74.7)$ & \\
\hline $6-7$ & $485(23.1)$ & $217(20.8)$ & $268(25.3)$ & \\
\hline $5-6$ & $0(0.0)$ & $0(0.0)$ & $0(0.0)$ & \\
\hline$<5$ & $0(0.0)$ & $0(0.0)$ & $0(0.0)$ & 0.017 \\
\hline \multicolumn{5}{|c|}{ Habitual sleep efficiency (\%) } \\
\hline$>85$ & $1716(81.7)$ & $861(82.7)$ & $855(80.7)$ & \\
\hline $75-84$ & $254(12.1)$ & $114(11.0)$ & $140(13.2)$ & \\
\hline $65-74$ & $80(3.8)$ & $39(3.7)$ & $41(3.9)$ & \\
\hline$<65$ & $50(2.4)$ & $27(2.6)$ & $23(2.2)$ & 0.408 \\
\hline \multicolumn{5}{|l|}{ Sleep disturbances } \\
\hline 0 & $193(9.2)$ & $124(11.9)$ & $69(6.5)$ & \\
\hline$<1 /$ week & $1717(81.8)$ & $841(80.8)$ & $876(82.7)$ & \\
\hline 1-2/week & $182(8.7)$ & $72(6.9)$ & $110(10.4)$ & \\
\hline$\geq 3 /$ week & $8(0.4)$ & $4(0.4)$ & $4(0.4)$ & $<0.001$ \\
\hline \multicolumn{5}{|c|}{ Use of sleep medication } \\
\hline 0 & 1959 (93.3) & $962(92.4)$ & $997(94.1)$ & \\
\hline$<1 /$ week & $97(4.6)$ & $51(4.9)$ & $46(4.3)$ & \\
\hline $1-2 /$ week & $35(1.7)$ & $23(2.2)$ & $12(1.1)$ & \\
\hline$\geq 3 /$ week & $9(0.4)$ & $5(0.5)$ & $4(0.4)$ & 0.231 \\
\hline \multicolumn{5}{|l|}{ Daytime dysfunction } \\
\hline Not a problem & $549(26.1)$ & $291(28.0)$ & $258(24.4)$ & \\
\hline Fairly a problem & 1089 (51.9) & $535(51.4)$ & $554(52.3)$ & \\
\hline Problem & 406 (19.3) & $197(18.9)$ & $209(19.7)$ & \\
\hline Very big problem & $56(2.7)$ & $18(1.7)$ & $38(3.6)$ & 0.022 \\
\hline \multicolumn{5}{|l|}{ Sleep quality } \\
\hline Good & $1310(62.4)$ & $684(65.2)$ & $626(59.1)$ & \\
\hline Poor & $790(37.6)$ & $357(34.8)$ & $433(40.9)$ & $<0.001$ \\
\hline \multicolumn{5}{|l|}{ Physical activity } \\
\hline Sufficient & $1626(77.4)$ & $890(85.5)$ & $736(69.5)$ & \\
\hline Insufficient & $474(22.6)$ & $151(14.5)$ & $323(30.5)$ & $<0.001$ \\
\hline \multicolumn{5}{|l|}{ Self-rated health } \\
\hline Good & 1935 (92.1) & 991 (95.2) & $944(89.1)$ & \\
\hline
\end{tabular}


Table 1 Continued

\begin{tabular}{|c|c|c|c|c|}
\hline & $\begin{array}{l}\text { Total sample } \\
(\mathrm{n}=\mathbf{2 1 0 0})\end{array}$ & $\begin{array}{l}\begin{array}{l}\text { Men } \\
(n=1041)\end{array} \\
\end{array}$ & $\begin{array}{l}\text { Women } \\
(n=1059)\end{array}$ & \\
\hline Study variables & $\mathrm{n}(\%)$ & n (\%) & n (\%) & P values* \\
\hline Poor & 165 (7.9) & $50(4.8)$ & 115 (10.9) & $<0.001$ \\
\hline \multicolumn{5}{|l|}{ Body mass index } \\
\hline Normal & $1706(81.2)$ & 769 (73.9) & 937 (88.5) & \\
\hline Overweight/obesity & $394(18.8)$ & $272(26.1)$ & $122(11.5)$ & $<0.001$ \\
\hline \multicolumn{5}{|l|}{ Life satisfaction } \\
\hline Good & 1951 (92.9) & 978 (93.9) & 973 (91.9) & \\
\hline Poor & $149(7.1)$ & $63(6.1)$ & $86(8.1)$ & 0.074 \\
\hline \multicolumn{5}{|l|}{ Socioeconomic status } \\
\hline Below average & $52(2.5)$ & $29(2.8)$ & $23(2.2)$ & \\
\hline Average & $1743(83.0)$ & 817 (78.5) & $926(87.4)$ & \\
\hline Above average & $305(14.5)$ & $195(18.7)$ & $110(10.4)$ & $<0.001$ \\
\hline \multicolumn{5}{|l|}{ Smoking status } \\
\hline No smoker & $1479(70.4)$ & 759 (72.9) & $720(68.0)$ & \\
\hline Past smoker & $115(5.5)$ & $53(5.1)$ & $62(5.8)$ & \\
\hline Current smoker & $506(24.1)$ & $229(22.0)$ & $277(26.1)$ & 0.032 \\
\hline \multicolumn{5}{|l|}{ Binge drinking } \\
\hline No & $1530(72.9)$ & $801(76.9)$ & $729(68.8)$ & \\
\hline Yes & $570(27.1)$ & $240(23.1)$ & $330(31.2)$ & $<0.001$ \\
\hline \multicolumn{5}{|l|}{ Chronic disease/s } \\
\hline No & $1905(90.7)$ & $943(90.6)$ & 962 (92.4) & \\
\hline Yes & 195 (9.3) & $98(9.4)$ & $97(7.6)$ & 0.438 \\
\hline \multicolumn{5}{|l|}{ Psychological distress } \\
\hline Low & $1878(89.4)$ & 970 (93.2) & 908 (85.7) & \\
\hline High & 222 (10.6) & $71(6.8)$ & $171(14.3)$ & $<0.001$ \\
\hline
\end{tabular}

${ }^{*} \mathrm{X}^{2}$ test.

using no sleep medication $(\mathrm{p}<0.001)$, having no problem with daytime dysfunction $(\mathrm{p}<0.001)$ and having good sleep quality $(\mathrm{p}<0.001)$ compared with 'insufficiently' active participants.

Basic descriptive statistics showing the prevalence of sleeping characteristics according to the level of physical activity are presented in table 2 . In general, higher percentage of 'sufficiently' active participants reported very good subjective sleep quality $(p<0.001), \leq 15 \mathrm{~min}$ of sleep latency $(\mathrm{p}<0.001)$, sleeping $>7$ hours per night $(p<0.001)$, having higher percentage of habitual sleep efficiency $(p=0.002)$, having no sleep disturbances $(p<0.001)$ and using no sleep medication $(p<0.001)$, having no problem with daytime dysfunction $(\mathrm{p}<0.001)$ and having 'good' sleep quality $(\mathrm{p}<0.001)$ compared with 'insufficiently active' participants.

Table 3 shows the relationships between all variables included in the analyses. Spearman's correlation coefficients between sleep quality and its domains were high $(\mathrm{r}=0.26-0.53)$ and roughly all variables entered as covariates were significantly related with sleep quality domains and sleep quality in general $(\mathrm{p}<0.05)$.

The associations between sleep quality domains with physical activity are presented in table 4 . In univariate model, poorer subjective sleep quality, higher sleep latency, shorter sleep duration, lower habitual sleep efficiency, use of sleep medication, increased daytime dysfunction and poor sleep quality were associated with 'insufficient' physical activity. In multivariate model, these associations remained significant after adjustment for gender, body mass index, self-rated health, life satisfaction, socioeconomic status, smoking status, alcohol consumption, presence of chronic diseases and psychological distress with the exception of sleep disturbances.

\section{DISCUSSION}

The main purpose of the present study was to explore the associations between 'poor' sleep quality and its domains and 'insufficient' physical activity in a large 
Open access

Table 2 Sleep characteristics according to the level of physical activity in the study participants (Croatia, 2017)

\begin{tabular}{|c|c|c|c|c|}
\hline \multirow[b]{2}{*}{ Study variables } & \multirow{2}{*}{$\begin{array}{c}\begin{array}{l}\text { Total sample } \\
(\mathrm{n}=2100)\end{array} \\
\mathrm{n}(\%)\end{array}$} & \multirow{2}{*}{$\begin{array}{l}\text { 'Sufficiently active' } \\
(\mathrm{n}=1626)\end{array}$} & \multirow{2}{*}{$\begin{array}{l}\text { 'Insufficiently active' } \\
\text { ( } \mathrm{n}=474)\end{array}$} & \multirow[b]{2}{*}{ P values* } \\
\hline & & & & \\
\hline \multicolumn{5}{|c|}{ Subjective sleep quality } \\
\hline Very good & $687(32.7)$ & $565(34.7)$ & $122(25.7)$ & \\
\hline Fairly good & $1179(56.1)$ & $903(55.5)$ & $276(58.2)$ & \\
\hline Fairly bad & $204(9.7)$ & $140(8.6)$ & $64(13.5)$ & \\
\hline Very bad & $30(1.4)$ & $18(1.1)$ & $12(2.5)$ & $<0.001$ \\
\hline \multicolumn{5}{|l|}{ Sleep latency (min) } \\
\hline$\leq 15$ & $292(13.9)$ & $242(14.9)$ & 50 (10.5) & \\
\hline $16-30$ & $868(41.3)$ & $694(42.7)$ & $174(36.7)$ & \\
\hline $31-60$ & $769(36.6)$ & 575 (35.2) & $197(41.6)$ & \\
\hline$>60$ & $171(8.1)$ & $118(7.3)$ & 53 (11.2) & $<0.001$ \\
\hline \multicolumn{5}{|c|}{ Sleep duration (hours) } \\
\hline$>7$ & $1615(76.9)$ & $1281(78.8)$ & $334(70.5)$ & \\
\hline $6-7$ & $485(23.1)$ & $345(21.2)$ & $140(29.5)$ & \\
\hline $5-6$ & $0(0.0)$ & $0(0.0)$ & $0(0.0)$ & \\
\hline$<5$ & $0(0.0)$ & $0(0.0)$ & $0(0.0)$ & $<0.001$ \\
\hline \multicolumn{5}{|c|}{ Habitual sleep efficiency (\%) } \\
\hline$>85$ & $1716(81.7)$ & $1350(83.0)$ & $366(77.2)$ & \\
\hline $75-84$ & $254(12.1)$ & $192(11.8)$ & $62(13.1)$ & \\
\hline $65-74$ & $80(3.8)$ & $53(3.3)$ & $27(5.7)$ & \\
\hline$<65$ & $50(2.4)$ & $31(1.9)$ & $19(4.0)$ & 0.002 \\
\hline \multicolumn{5}{|l|}{ Sleep disturbances } \\
\hline 0 & $193(9.2)$ & $146(9.0)$ & $47(9.9)$ & \\
\hline$<1 /$ week & $1717(81.8)$ & $1355(83.3)$ & $362(76.4)$ & \\
\hline $1-2 /$ week & $182(8.7)$ & $122(7.5)$ & $60(12.7)$ & \\
\hline$\geq 3 /$ week & $8(0.4)$ & $3(0.2)$ & $5(1.1)$ & $<0.001$ \\
\hline \multicolumn{5}{|c|}{ Use of sleep medication } \\
\hline 0 & 1959 (93.3) & $1539(94.6)$ & $420(88.6)$ & \\
\hline$<1 /$ week & $97(4.6)$ & $64(3.9)$ & $33(7.0)$ & \\
\hline $1-2 /$ week & $35(1.7)$ & $20(1.2)$ & $15(3.2)$ & \\
\hline$\geq 3 /$ week & $9(0.4)$ & $3(0.2)$ & $6(1.3)$ & $<0.001$ \\
\hline \multicolumn{5}{|l|}{ Daytime dysfunction } \\
\hline Not a problem & $549(26.1)$ & $439(27.0)$ & $110(23.2)$ & \\
\hline Fairly a problem & $1089(51.9)$ & $865(53.2)$ & $224(47.3)$ & \\
\hline Problem & 406 (19.3) & $289(17.8)$ & $117(24.7)$ & \\
\hline Very big problem & $56(2.7)$ & $33(2.0)$ & $23(4.9)$ & $<0.001$ \\
\hline \multicolumn{5}{|l|}{ Sleep quality } \\
\hline Good & $1310(62.4)$ & 1065 (81.3) & $245(18.7)$ & \\
\hline Poor & $790(37.6)$ & $561(71.0)$ & $229(29.0)$ & $<0.001$ \\
\hline \multicolumn{5}{|l|}{ Gender } \\
\hline Men & $1041(49.6)$ & $890(54.7)$ & $151(31.9)$ & \\
\hline Women & $1059(50.4)$ & $736(45.3)$ & $323(68.1)$ & $<0.001$ \\
\hline \multicolumn{5}{|l|}{ Self-rated health } \\
\hline Good & $1935(92.1)$ & 1509 (92.8) & $426(89.9)$ & \\
\hline
\end{tabular}


Table 2 Continued

\begin{tabular}{|c|c|c|c|c|}
\hline & $\begin{array}{l}\text { Total sample } \\
(\mathrm{n}=2100)\end{array}$ & $\begin{array}{l}\text { 'Sufficiently active' } \\
(\mathrm{n}=1626)\end{array}$ & $\begin{array}{l}\text { 'Insufficiently active' } \\
(\mathrm{n}=474)\end{array}$ & \\
\hline Study variables & $\mathrm{n}(\%)$ & n (\%) & n (\%) & $P$ values* \\
\hline Poor & $165(7.9)$ & $117(7.2)$ & $48(10.1)$ & 0.025 \\
\hline \multicolumn{5}{|l|}{ Body mass index } \\
\hline Normal & $1706(81.2)$ & $1316(81.9)$ & $390(82.2)$ & \\
\hline Overweight/obesity & 394 (18.8) & $310(19.1)$ & $84(17.7)$ & 0.510 \\
\hline \multicolumn{5}{|l|}{ Life satisfaction } \\
\hline Good & 1951 (92.9) & 1521 (93.5) & $430(90.7)$ & \\
\hline Poor & $149(7.1)$ & $105(6.5)$ & $44(9.3)$ & 0.025 \\
\hline \multicolumn{5}{|l|}{ Socioeconomic status } \\
\hline Below average & $52(2.5)$ & $41(2.5)$ & $11(2.3)$ & \\
\hline Average & $1743(83.0)$ & $1333(82.0)$ & $410(86.5)$ & \\
\hline Above average & 305 (14.5) & $252(15.5)$ & $53(11.2)$ & 0.007 \\
\hline \multicolumn{5}{|l|}{ Smoking status } \\
\hline No smoker & $1479(70.4)$ & $1161(71.4)$ & $318(67.1)$ & \\
\hline Past smoker & $115(5.5)$ & $85(5.2)$ & $30(6.3)$ & \\
\hline Current smoker & $506(24.1)$ & $380(23.4)$ & $126(26.6)$ & 0.160 \\
\hline \multicolumn{5}{|l|}{ Binge drinking } \\
\hline No & $1530(72.9)$ & $1202(73.9)$ & $328(69.2)$ & \\
\hline Yes & $570(27.1)$ & $424(26.1)$ & $146(30.8)$ & 0.330 \\
\hline \multicolumn{5}{|l|}{ Chronic disease/s } \\
\hline No & 1905 (90.7) & 1485 (91.3) & 420 (89.2) & \\
\hline Yes & 195 (9.3) & $141(8.7)$ & $54(11.5)$ & 0.537 \\
\hline \multicolumn{5}{|l|}{ Psychological distress } \\
\hline Low & $1878(89.4)$ & $1480(91.0)$ & $398(84.0)$ & \\
\hline High & $222(10.6)$ & $146(9.0)$ & 76 (16.0) & $<0.001$ \\
\hline
\end{tabular}

${ }^{*} \mathrm{X}^{2}$ test.

sample of young adults. Our study showed that after adjusting for numerous covariates, poorer subjective sleep quality, higher sleep latency, shorter sleep duration, lower habitual sleep efficiency, use of sleep medication, increased daytime dysfunction and poor sleep quality were associated with 'insufficient' physical activity.

Our results are in line with previous studies aimed to explore the associations between sleep quality and physical activity. ${ }^{70-12} 2728$ Specifically, a longitudinal study conducted among older adults and aiming to explore the bidirectional associations between sleep quality and physical activity showed that individuals who experienced 'good' sleep quality and had no sleeping problems had more energy to engage in physical activity compared with those individuals with 'poor' sleep quality. ${ }^{11}$ Another study conducted among a large sample of National Guard ( $n=14148 ; 83.4 \%$ men) showed that 'poor' sleepers were more likely to report 'poor' health, be overweight or obese and were in the lowest quartile of the emotional, social, family and fitness dimensions. Moreover, 'poor' sleepers were significantly less likely to meet aerobic exercise and resistance training recommendations in terms of passing army physical fitness test in the highest quartile. ${ }^{10}$ Previous studies have also shown that 'poor' sleep is associated with a decrease in maximal oxygen uptake, ${ }^{29}$ and that chronic and acute sleep loss is associated with exercise-related injuries, ${ }^{30}$ leading to decreased engagement in physical activity. The association between sleep deprivation and increased exercise-related injuries is driven to the decrease of proprioception and postural control enabling full functional recovery of the muscles after exercise training. ${ }^{12}$

Interestingly, our results showed that after adjusting for numerous sociodemographic, lifestyle and psychological covariates, increased level of sleep disturbances was not significantly associated with 'insufficient' physical activity, which is in line with previous studies. ${ }^{31}$ Specifically, Chang $e t a l^{31}$ found that moderate physical activity was not significantly associated with insomnia symptoms. On the other hand, the same study showed that more time spent in vigorous physical activity was significantly associated with decreased insomnia symptoms. In general, one recent 


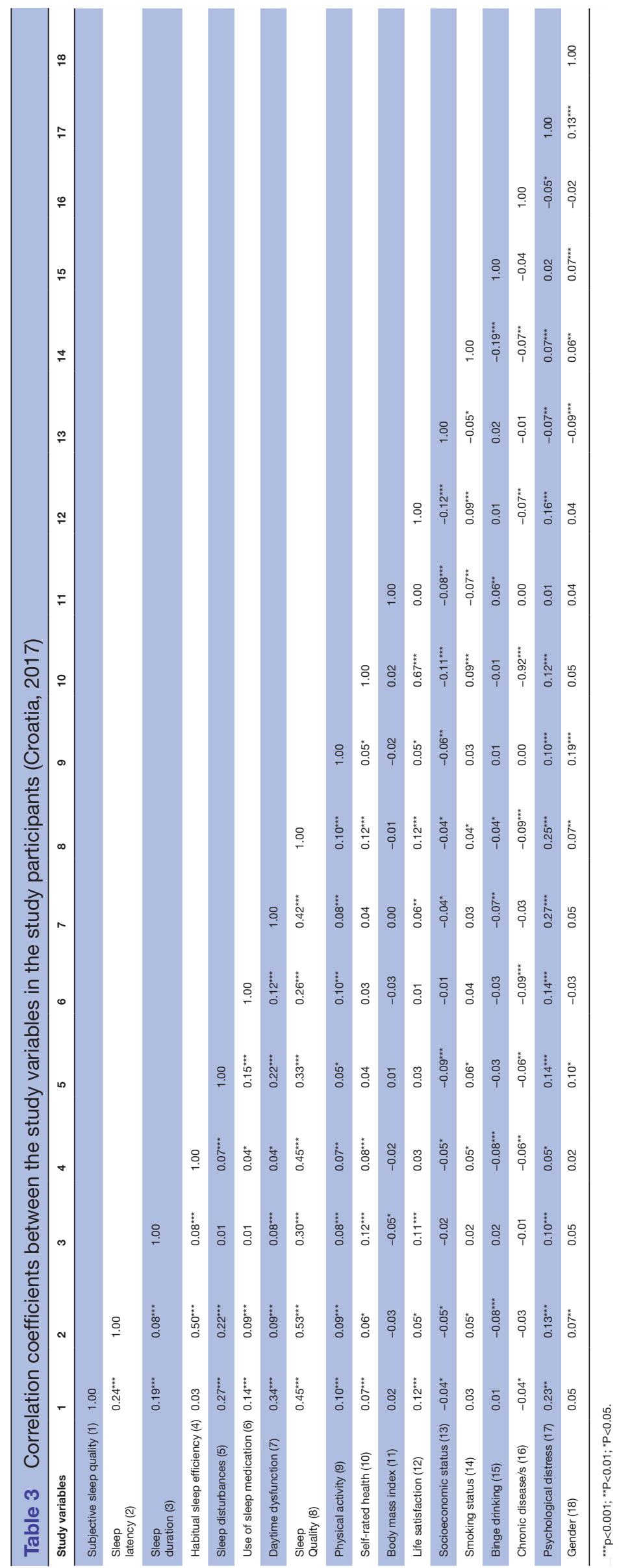

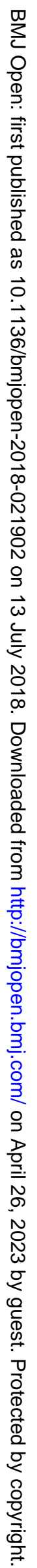


Table 4 ORs for insufficient physical activity in study participants (Croatia, 2017)

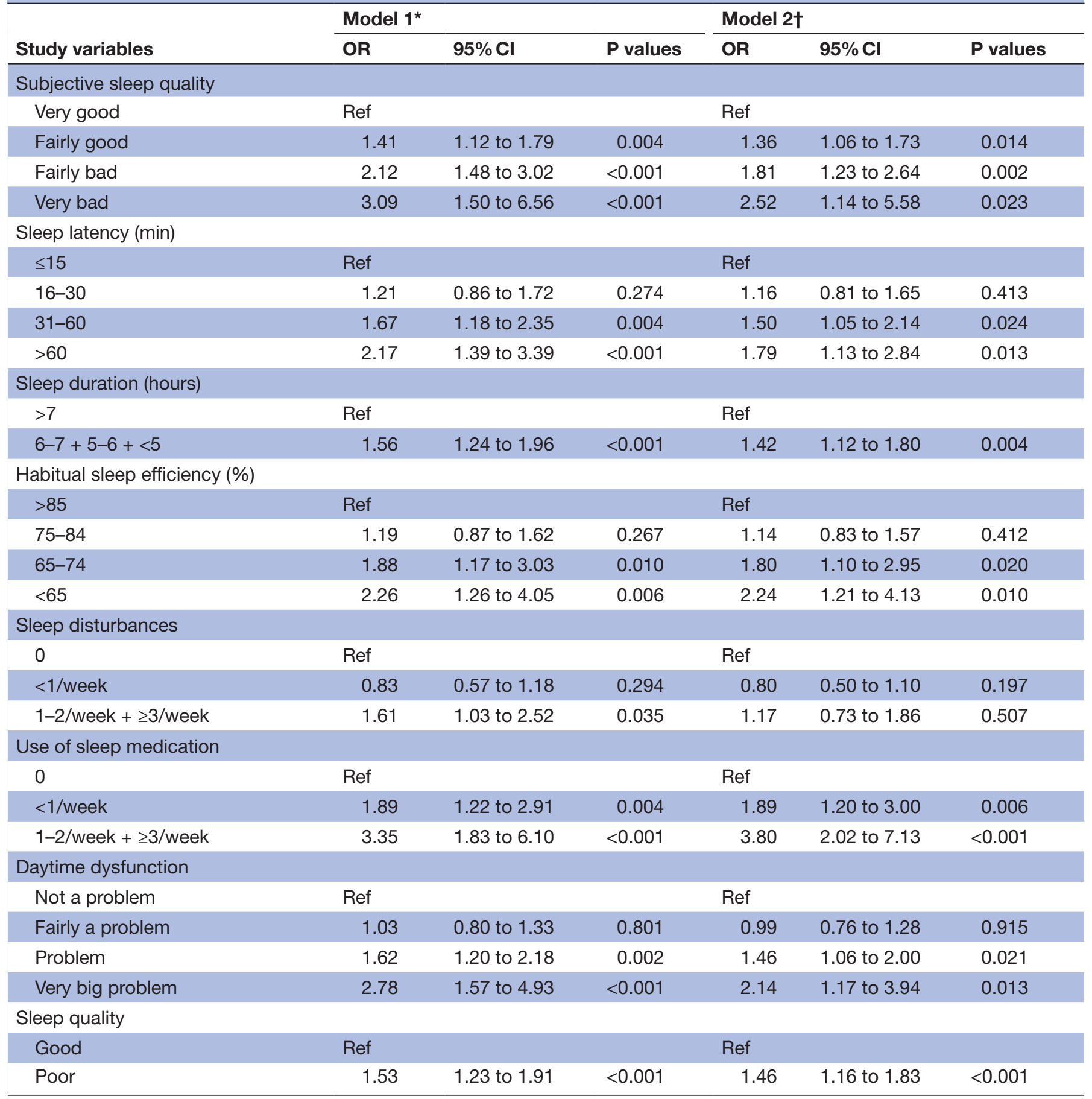

*Examine the associations between sleep quality and insufficient physical activity.

$\dagger$ Examine the associations between sleep quality and insufficient physical activity after adjusting for gender, body mass index, self-rated health, life satisfaction, socioeconomic status, smoking status, binge drinking, presence of chronic disease/s and psychological distress.

systematic review showed that although a significant scientific effort has been made to explore the associations between sleep quality (including sleep disturbances) and physical activity, the physiological and psychological mechanisms remain unclear with conflicting results. ${ }^{32}$ However, the lack of significant association between sleep disturbances and 'insufficient' physical activity could be explained by using self-reports to assess the level of physical activity and sleep disturbances, which might have led to different perception and method bias. Also, a relatively small number of participants in our study reported experiencing some kind of sleep disturbances $(9.1 \%)$ and $22.6 \%$ of the whole sample was categorised as insufficiently' active, which could have led to unstable parameter estimates (extreme 95\% interval values). Moreover, we performed the study on university students, limiting the generalisability of our findings to other age groups. Although not statistically significant, our results showed 
that participants experiencing sleep disturbances were $17 \%$ more likely to be 'insufficiently' physically active compared with the participants with no sleep disturbances complaining.

This study has several strengths. First, we conducted a study among a large sample of young adults. Second, we used previously validated questionnaires to assess physical activity and sleep quality. Moreover, we adjusted for numerous covariates (ie, gender, body mass index, self-rated health, life satisfaction, socioeconomic status, smoking status, alcohol consumption and presence of chronic diseases), which could affect both physical activity and sleep quality. Third, we additionally adjusted for psychological distress. As pointed out by one previous study, studies have failed to control for variables such as psychiatric symptoms to exclude plausible alternative explanations. ${ }^{6}$

However, our study has some limitations. First, we used a cross-sectional design, so we cannot exclude the possibility of reverse causality, that is, 'insufficient' physical activity led to 'poor' sleep quality. Previous studies have confirmed such associations. ${ }^{12}$ Specifically, Chennaoui et al showed that physical activity promotes and serves energy conservation, body restoration and thermoregulatory functions, and that physical activity even done before bedtime impacts positively on sleep. ${ }^{12}$ However, based on our findings, sleep quality were associated with physical activity, yet the direction of association remained unknown. Second, we used subjective measures to assess physical activity and sleep quality. Self-report measures tend to lead to considerable measurement error, recall bias and social desirability effect. ${ }^{33}$ Third, we did not collect any information about physiological (hormonal) parameters nor daylight exposure, while daylight exposure has a beneficial effect on well-being and psychological functioning. ${ }^{6}$ Fourth, we based our sample on university students situated in only one city, and including other cities in the study could have potentially led to different results. University students do not represent young adults in general and sleep quality domains and physical activity might be different in other populations. Thus, future studies exploring and tracking the associations between sleep quality and physical activity level in other populations using objective measures (actigraphy) are warranted.

\section{CONCLUSIONS}

Our study showed that after adjusting for gender, body mass index, self-rated health, life satisfaction, socioeconomic status, smoking status, alcohol consumption, presence of chronic diseases and psychological distress, 'poor' subjective sleep quality, 'higher' sleep latency, 'shorter' sleep duration, 'lower' percentage of habitual sleep efficiency, 'use' of sleep medication, 'having problem' with daytime dysfunction and 'poor' sleep quality were associated with 'insufficient' physical activity. Future studies using objective methods to assess physical activity (actigraphy) and sleep quality (polysomnography) over a longer period of follow-up should be performed in order to establish causal associations between sleep quality and physical activity.

Acknowledgements We thank Breda Berić and Andro Štefan for data collection. Also, we thank the students for their enthusiastic participation in the study.

Contributors LŠ, GS, TK and DK conceived the study, wrote the manuscript and analysed the data. All authors reviewed the final version of the manuscript.

Funding This paper was self-funded.

Competing interests None declared.

Patient consent Obtained.

Ethics approval Institutional Ethics Committee of the Faculty of Kinesiology, University of Zagreb, Croatia.

Provenance and peer review Not commissioned; externally peer reviewed.

Data sharing statement All the data are freely available.

Open access This is an open access article distributed in accordance with the Creative Commons Attribution Non Commercial (CC BY-NC 4.0) license, which permits others to distribute, remix, adapt, build upon this work non-commercially, and license their derivative works on different terms, provided the original work is properly cited, appropriate credit is given, any changes made indicated, and the use is non-commercial. See: http://creativecommons.org/licenses/by-nc/4.0/.

\section{REFERENCES}

1. World Health Organization. Global recommendations on physical activity for health. Geneva, Switzerland: WHO Press, 2010.

2. Dumith SC, Hallal PC, Reis RS, et al. Worldwide prevalence of physical inactivity and its association with human development index in 76 countries. Prev Med 2011;53:24-8.

3. Warburton DE, Nicol CW, Bredin SS. Health benefits of physical activity: the evidence. CMAJ 2006;174:801-9.

4. Bauman AE, Reis RS, Sallis JF, et al. Correlates of physical activity: why are some people physically active and others not? Lancet 2012;380:258-71.

5. Gerber M, Brand S, Holsboer-Trachsler E, et al. Fitness and exercise as correlates of sleep complaints: is it all in our minds? Med Sci Sports Exerc 2010;42:893-901.

6. Youngstedt SD, Freelove-Charton JD. Exercise and sleep. In: Faulkner GEJ, Taylor AH, eds. Exercise, health and mental health. London (UK): Routledge, 2005.

7. Landry GJ, Best JR, Liu-Ambrose T. Measuring sleep quality in older adults: a comparison using subjective and objective methods. Front Aging Neurosci 2015;7:166.

8. Brand S, Kalak N, Gerber M, et al. High self-perceived exercise exertion before bedtime is associated with greater objectively assessed sleep efficiency. Sleep Med 2014;15:1031-6.

9. Buman MP, Phillips BA, Youngstedt SD, et al. Does nighttime exercise really disturb sleep? Results from the 2013 National Sleep Foundation Sleep in America Poll. Sleep Med 2014;15:755-61.

10. Lentino CV, Purvis DL, Murphy KJ, et al. Sleep as a component of the performance triad: the importance of sleep in a military population. US Army Med Dep J 2013:98-108.

11. Holfeld B, Ruthig JC. A longitudinal examination of sleep quality and physical activity in older adults. J App/ Gerontol 2014;33:791-807.

12. Chennaoui M, Arnal PJ, Sauvet F, et al. Sleep and exercise: a reciprocal issue? Sleep Med Rev 2015;20:59-72.

13. Keyes KM, Maslowsky J, Hamilton A, et al. The great sleep recession: changes in sleep duration among US adolescents, 19912012. Pediatrics 2015;135:460-8.

14. Keating XD, Guan J, Piñero JC, et al. A meta-analysis of college students' physical activity behaviors. J Am Coll Health 2005;54:116-26.

15. Fatima Y, Doi SA, Najman JM, et al. Exploring gender difference in sleep quality of young adults: findings from a large population study. Clin Med Res 2016;14:138-44.

16. Lindberg E, Janson C, Gislason T, et al. Sleep disturbances in a young adult population: can gender differences be explained by differences in psychological status? Sleep 1997;20:381-7.

17. Krističević T, Štefan L. Goran Sporiš. The Associations between Sleep Duration and Sleep Quality with Body-Mass Index in a 
Large Sample of Young Adults. Int J Environ Res Public Health 2018:15:758

18. Štefan L, Sporiš G, Krističević T. Are lower levels of physical activity and self-rated fitness associated with higher levels of psychological distress in Croatian young adults? A cross-sectional study. PeerJ 2018:6:e4700.

19. Štefan L, Krističević T, Sporiš G. The associations of selfreported physical fitness and physical activity with sleep quality in young adults: A population-based study. Ment Health Phys Act 2018;14:131-5.

20. Craig CL, Marshall AL, Sjöström M, et al. International physical activity questionnaire: 12 -country reliability and validity. Med Sci Sports Exerc 2003;35:1381-95.

21. Buysse DJ, Reynolds CF, Monk TH, et al. The Pittsburgh Sleep Quality Index: a new instrument for psychiatric practice and research. Psychiatry Res 1989;28:193-213.

22. Larsson D, Hemmingsson T, Allebeck $P$, et al. Self-rated health and mortality among young men: what is the relation and how may it be explained? Scand J Public Health 2002;30:259-66.

23. Štefan L, Juranko D, Prosoli R, et al. Self-reported sleep duration and self-rated health in young adults. J Clin Sleep Med 2017;13:899-904.

24. Peltzer K, Pengpid S. Sleep duration and health correlates among university students in 26 countries. Psychol Health Med 2016;21:208-20.
25. Zhi TF, Sun XM, Li SJ, Sj L, et al. Associations of sleep duration and sleep quality with life satisfaction in elderly Chinese: The mediating role of depression. Arch Gerontol Geriatr 2016;65:211-7.

26. Kessler RC, Barker PR, Colpe LJ, et al. Screening for serious mental illness in the general population. Arch Gen Psychiatry 2003;60:184-9.

27. Lee AJ, Lin WH. Association between sleep quality and physical fitness in female young adults. J Sports Med Phys Fitness 2007;47:462-7.

28. Chang SP, Chen YH. Relationships between sleep quality, physical fitness and body mass index in college freshmen. J Sports Med Phys Fitness 2015;55:1234-41.

29. Chen HI. Effects of 30-h sleep loss on cardiorespiratory functions at rest and in exercise. Med Sci Sports Exerc 1991;23:193???198-8.

30. Dattilo M, Antunes HK, Medeiros A, et al. Sleep and muscle recovery: endocrinological and molecular basis for a new and promising hypothesis. Med Hypotheses 2011;77:220-2.

31. Chang JJ, Pien GW, Stamatakis KA, et al. Association between physical activity and insomnia symptoms in rural communities of southeastern Missouri, Tennessee, and Arkansas. J Rural Health 2013;29:239-47.

32. Fullagar HH, Skorski S, Duffield R, et al. Sleep and athletic performance: the effects of sleep loss on exercise performance, and physiological and cognitive responses to exercise. Sports Med 2015;45:161-86.

33. Shephard RJ. Limits to the measurement of habitual physical activity by questionnaires. Br J Sports Med 2003;37:197-206. 